\title{
Multiferroic based 2D phononic crystals: Band structure and wave propagations
}

\author{
Selami Palaz, Zafer Ozer, Cengiz Ahundov, Amirullah M. Mamedov \& Ekmel \\ Ozbay
}

To cite this article: Selami Palaz, Zafer Ozer, Cengiz Ahundov, Amirullah M. Mamedov \& Ekmel Ozbay (2019) Multiferroic based 2D phononic crystals: Band structure and wave propagations, Ferroelectrics, 544:1, 88-95, DOI: 10.1080/00150193.2019.1598193

To link to this article: https://doi.org/10.1080/00150193.2019.1598193

册 Published online: 16 Aug 2019.

Submit your article to this journal

山 Article views: 46

Q View related articles $₫$

View Crossmark data 


\title{
Multiferroic based 2D phononic crystals: Band structure and wave propagations
}

\author{
Selami Palaz ${ }^{a}$, Zafer Ozer ${ }^{b}$, Cengiz Ahundovc, Amirullah M. Mamedov ${ }^{c, d}$, and \\ Ekmel Ozbay ${ }^{d}$ \\ ${ }^{a}$ Department of Physics, Faculty of Sciences, Harran University, Sanliurfa, Turkey; ${ }^{b}$ Mersin Vocational \\ High School, Mersin University, Mersin, Turkey; 'International Scientific Center, Baku State University, \\ Baku, Azerbaijan; ${ }^{d}$ Nanotechnology Research Center, Bilkent University, Bilkent, Ankara, Turkey
}

\begin{abstract}
In the present work the acoustic band structure of a two-dimensional phononic crystal containing an organic ferroelectric (PVDFpolyvinylidene fluoride) and muliferroic material $\left(\mathrm{LiVCuO}_{4}\right)$ were investigated by the plane-wave-expansion method. A two-dimensional PC with square lattices composed of $\mathrm{LiVCuO}_{4}$ cylindrical rods embedded in the PVDF matrix are studied to find the existence of stop bands for the waves of certain energy. This phononic bandgap - forbidden frequency range - allows sound to be controlled in many useful ways in structures that can act as sonic filters, waveguides or resonant cavities. Phononic band diagram $\omega=\omega(\mathbf{k})$ for a $2 \mathrm{D} P C$, in which non-dimensional frequencies $\omega \mathrm{a} / 2 \pi \mathrm{c}$ (c-velocity of wave) were plotted versus the wavevector $\mathrm{k}$ along the $\Gamma-\mathrm{X}-\mathrm{M}-\Gamma$ path in the square Brillouin zone show four stop bands in the frequency range $0.01-8.0 \mathrm{kHz}$. The ferroelectric properties of PVDF and unusual properties of multiferroic $\mathrm{LiVCuO}_{4}$ give us the ability to control the wave propagation through the PC in over a wide frequency range.
\end{abstract}

\section{ARTICLE HISTORY}

Received 25 June 2018

Accepted 23 December 2018

\section{KEYWORDS}

Phononic crystals; acoustic band gaps; multiferroic; wave propagation

\section{Introduction}

Composite structures comprising periodic arrangements of scatterers embedded in a host matrix material can strongly affect the propagation of classical waves, such as electromagnetic waves, acoustic waves, and elastic waves. Composite materials that modulate the propagation characteristics of electromagnetic waves are classified as photonic crystals, whereas the term phononic crystal (PC) describes composites affecting acoustic wave propagation or elastic wave propagation. Since the first demonstration of a photonic crystal in 1987 [1, 2], the study of periodic media has steadily increased in popularity. PCs come in one-dimensional (1D), two-dimensional (2D), and three-dimensional (3D) forms. 1D PCs consist of alternating layers or stacks of different elastic materials. 2D PCs comprise periodic arrays of rod-like inclusions of one elastic material dispersed throughout a host matrix medium of a different elastic material. 3D PCs utilize lattice arrangements (simple cubic, body-centered cubic, face-centered cubic) of elastic scatterers of various geometries (spheres, cubes, etc.) dispersed throughout a dissimilar host matrix material. 
The periodic nature of PCs allows for the modification of phonon dispersion via Bragg scattering, multiple scattering processes, and/or local resonances and offers a variety of properties such as spectral properties (x-space), wave vector properties (k-space), and phase properties (u-space) $[1,2]$. The most interesting aspect of such acoustic metamaterials arises from the presence of phononic band gaps (PBGs). The band gap in a phononic crystal is caused by a periodic variation in the density and changes of the speed of sound in the crystal. The band gaps exist in a certain range of frequencies where acoustic/elastic wave propagation is forbidden. In such a situation, the phononic crystal behaves as a perfect acoustic mirror and an incident wave is perfectly reflected outside for the rejected frequencies [2]. The incident waves with frequencies within the band gap are totally reflected by the phononic crystal. Phononic crystals, due to their unique feature of a band gap, have exciting applications in acoustic filters, acoustic wave guides and acoustic mirrors [2], and therefore they are of primary interest for communication technologies. These periodic structures are mainly designed to control the propagation of mechanical waves [2-9] or the focusing of elastic waves (gradient index PCs, acoustical super-lenses ... ). PCs can be considered as acoustic phononic crystals, and elastic phononic crystals. In acoustic phononic crystals, mechanical waves passing through a gas or a fluid are known as acoustic waves, while those passing through an elastic solid matrix are called elastic waves. The band structure of a phononic crystal can be controlled by changing the geometry of the inclusions [2,9], the volume fraction of inclusions [2], by varying lattice spacing and topology [2], or by varying the mechanical characteristics of the constitutive materials that change through the application of external stimuli $[2,9]$. Interestingly, some authors have proposed and demonstrated that large deformation may be used to tune the phononic band gaps of $2 \mathrm{D}$ periodic structures [2, 3, 9], and suggested the use of electrorheological materials in conjunction with the application of an external electric field [9], as well as the effect of temperature on the elastic moduli.[2]. However, some authors have also studied and analyzed the effects of piezoelectricity on the dispersion curves and they show that the change is negligible for small filling fractions, but significant for large ones [2].

$\mathrm{LiVCuO}_{4}$ is a multiferroic material where the ferroelectric and ferromagnetic properties are exhibited in a single phase [10]. Since magnetization cannot be controlled by an electric field in a single phase, the coupling between ferromagnetic and ferroelectric is so weak that it cannot contribute to the magnetic transition. Therefore, many multiferroics have a low Curie temperature. Ferromagnetism and ferroelectric loss occur due to weak coupling and a low Curie temperature. Therefore, multiferroic-ferrite composites can be synthesized as a result of a high Curie temperature and suitable ferromagnetic-ferroelectric coupling. As a result of the work done, it has been reported that the elastic coupling between multiferroics and spinel ferrites may be formed by epitaxial or layered alignment $[10,11]$. On the other hand, polyvinylidene fluoride (PVDF) is a well- known material for its toughness, stability, and ability to withstand long-term exposure to ultraviolet radiation [12]. The major advantage of using polymeric materials is that they have low weight, low thermal conductivity, high heat resistance, and mechanical flexibility. Moreover, the study of mechanical waves in gases, fluids, and solids including vibration has been demonstrated that polymerbased phononic metamaterials allow adaptive, reconfigurable, and flexible functionality in the acoustic and ultrasonic regimes. 
This paper theoretically and numerically investigates the propagation characteristics and the band structure of longitudinal waves propagating in a $2 \mathrm{D}$ phononic crystal composed of $\mathrm{LiVCuO}_{4}$ cylinders embedded in a PVDF matrix. More explicitly, we study the $2 \mathrm{D}$ composites by solving the basic acoustic wave equation and use Bloch wave analysis to identify the band gaps. The results obtained are based on the plane wave expansion (PWE) method [2].

\section{Model and the calculation method}

The PC is a $2 \mathrm{D}$ composite structure consisting of a square array of $\mathrm{LiVCuO}_{4}$ inclusions (cylinders) embedded in a host matrix of PVDF. The density, longitudinal speed of sound, and transverse speed of sound for PVDF are $1780 \mathrm{~kg} / \mathrm{m}^{3}, 2135 \mathrm{~m} / \mathrm{s}$, and $1300 \mathrm{~m} /$ s, respectively. For $\mathrm{LiVCuO}_{4}$, the parameters are $7850 \mathrm{~kg} / \mathrm{m}^{3}, 5825 \mathrm{~m} / \mathrm{s}$, and $2950 \mathrm{~m} / \mathrm{s}$. Spectral and wave vector properties for the infinite, periodic PC are evaluated using the PWE method [2]. Spectral, wave vector, and phase properties for a finite slab of the PC are evaluated using the FDTD method [2] For FDTD analysis, the PC slab is sandwiched between two homogeneous regions of material. Here we focus on the P-wave (pressure wave) or acoustic wave propagation through a periodic array of cylindrical scatterers. Although one of the media is a solid medium, we will assume the matrix as a solid medium to take the advantage of solid/solid approximation. Such an approximation is reasonable if we only consider that the waves propagate as longitudinal waves by means of adiabatic compression and decompression. The major difference between the approximations will mainly be observed for higher bands. In other words, this approximation can yield acceptable results for lower bands. Assuming a homogeneous and isotropic medium, we can separate the wave modes and use methods based on acoustic wave theory.

To calculate the band gaps for phononic crystals, different algorithms have been developed. These numerical methods include, the plane wave expansion method (PWE), transfer matrix method (TMM), finite difference time domain method (FDTD), and multiple scattering theory (MST) [2] method. Among them, the PWE method in the frequency domain and the FDTD method in time domain are the two that are used most widely. We will use the PWE method because of its simplicity. The advantage of the PWE method is that it must neglect the transverse mode existing in solid and simply treat the solid as an artificial "fluid". It must be noted that one of the advantages of PVDF like materials is that they can be fluid-processed as a melt. The considered system is a $2 \mathrm{D}$ phononic crystal composed of $\mathrm{LiVCuO}_{4}$ inclusions in a PVDF host in a square lattice. A square lattice formed of cylindrical rods has a lattice constant, a, is $0.05 \mathrm{~m}$ and the scatterers' radius, $\mathrm{r}_{0}$, is $0.025 \mathrm{~m}$. The calculation model of the studied system and its unit cell is shown in Figure 1.

The normalized frequencies $\omega$ are calculated by the PWE method. For the PWE method the solid matrix is considered as a fluid but with the material parameters of the actual PVDF. Under the assumptions that we made, the acoustic wave equation can be written as [2]:

$$
\left(C_{11}\right)^{-1} \frac{\partial^{2} p}{\partial t^{2}}=\vec{\nabla} \cdot\left(\rho^{-1} \nabla p\right)
$$




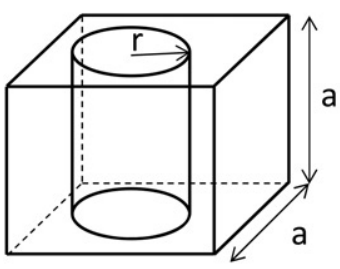

(a)

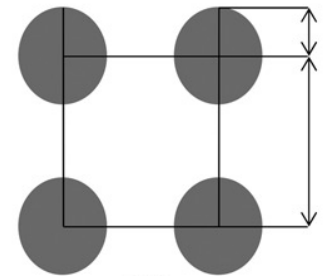

(b)

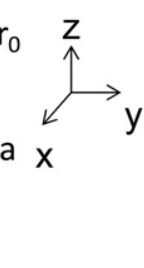

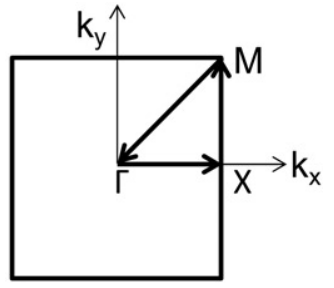

(c)

Figure 1. Unit cell of a square lattice two dimensional phononic crystal (a). Transverse cross-section of a representative 2-D phononic crystal (b) and Brillouin zone of 2-D square lattice PCs (c).

where $\mathrm{p}$ is the pressure wave, $\mathrm{C}_{11}$ is the longitud'nal velocity and $\rho$ is the mass density. The plane-wave expansion method has been applied in calculating the band gap of the system. The important part of the method is the structure factor $S(\mathbf{G})$, it is defined in two-dimensional system as

$$
S(\vec{G})=2 f J_{1}\left(G r_{0}\right) /\left(G r_{0}\right)
$$

where $A$ is the area of the unit cell, $a_{i}$ is the area of inclusions in the unit cell, $G$ is the two-dimensional reciprocal lattice vector. For cylindrical rods, the structure factor is where $\mathrm{f}$ is the filling fraction. The Fourier coefficients for $\mathrm{C}_{11}$ and $\rho$ can be expressed as

$$
F(\vec{G})= \begin{cases}F_{a}^{-1} f+F_{b}^{-1}(1-f)=\overline{F^{-1}} & \text { for } \vec{G}=0 \\ \left(F_{a}^{-1}-F_{b}{ }^{-1}\right) S(\vec{G})=\Delta\left(F^{-1}\right) S(\vec{G}) & \text { for } \vec{G} \neq 0\end{cases}
$$

where $F$ is the physical parameter used for $C_{11}$ and for $\rho$, and the subindex " $b$ " stands for the host material.

\section{Results and discussions}

The computational model based on the PWE method and the physical parameters of the system have been given in the above section. The numerical results for the band structure are expressed in terms of the normalized or reduced frequency $\Omega$, which is an expression of $\left(\omega a / 2 \pi c_{l}\right)$ and it ranges from 0 to 3.0. Figure 2 shows the calculated band structures of the $2 \mathrm{D}$ phononic crystal given in Figure 1. It is seen in Figure 2 that there exists complete acoustic band gaps (indicated by shaded region) for the assumed model around 0.4 to 2.8 . The complete band gap along the right-handed triangle $\Gamma-\mathrm{X}-\mathrm{M}-\Gamma$ with vertices at $\Gamma=(0,0), \mathrm{X}=(\pi, 0)$ and $\mathrm{M}=(\pi, \pi)$ show us that the entire angular range of the wave propagation direction, while other directions are not forbidden within a certain range of frequencies. The density of states as a function of frequency is also computed and the figure in the right side panel shows the corresponding phononic density of states (DOS) as a function of frequency. The phononic density of states were calculated by sampling $k$-points in the two dimensional BZ. We found the density of states as a function of frequency $\omega$ by doing a sum of all the bands over all the frequencies. DOS calculations are particularly important since some physical properties, such as thermal conductivity and specific heat, can be modified by changing the phonon density of states in a phononic crystal. Briefly, they provide insight into the microscopic and macroscopic properties. 


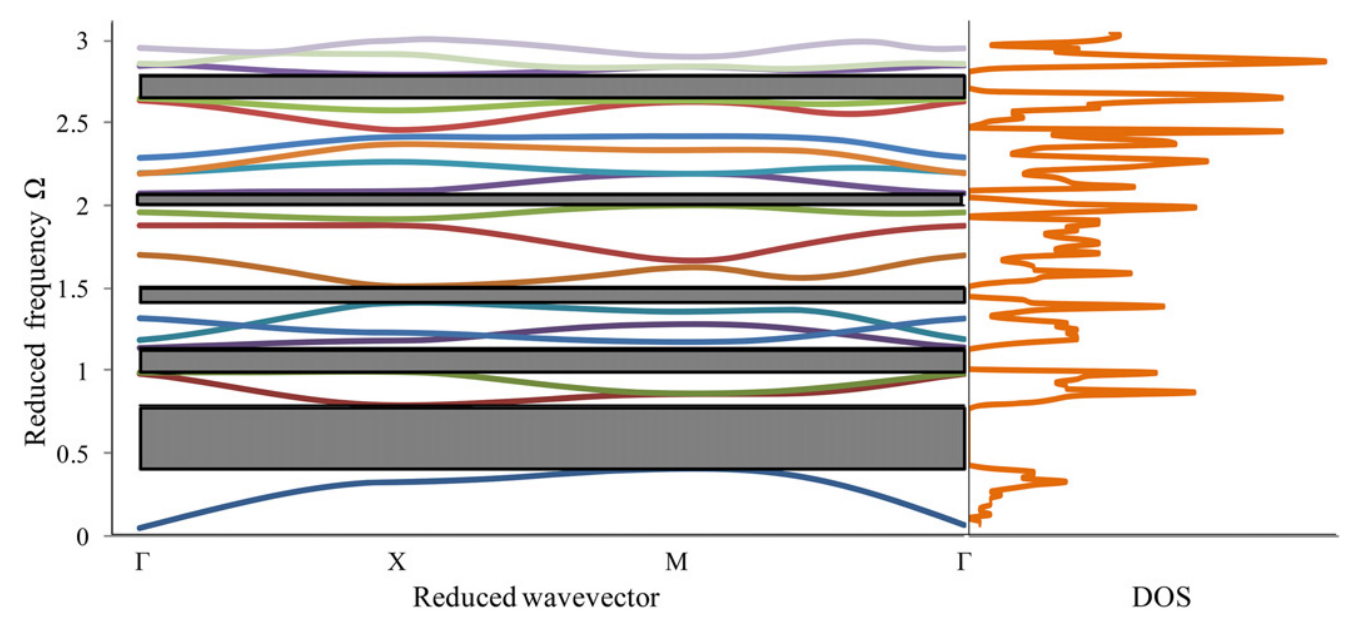

Figure 2. The band structure of the studied 2D PVDF/LiVCuO 4 s phononic crystal calculated in the first Brillouin zone.

The dispersion relation, $\omega(\mathrm{k})$, for the first and second bands were calculated. For this calculation, we have to calculate the full band diagram for all k-vectors in the first Brillouin zone, not like the band diagram that was calculated only along the $\Gamma-\mathrm{X}, \mathrm{X}-\mathrm{M}$, and $\mathrm{M}-\Gamma$ directions in the first Brillouin zone. Like the band structure construction, we used the frequency-domain PWE method to create the equi-frequency contours. They are illustrated in Figure 3. The plots provide a better understanding of how such dispersion influences acoustic velocity. Moreover, the contour plots of the associated dispersion surfaces are also shown in Figure 3. The equifrequency contours (EFC) are at the intersection of the 3D dispersion curves with a horizontal plane and they provide an essential source of information. Eigenfrequency contours are especially informative regarding their qualitative shape. By considering the acoustical wave vector and the group velocity as local parameters, the shape of the equifrequency contours can be used accurately to account for anisotropy. Figure 3 shows equi-frequency contours in reduced k space and they are mostly circular around the high symmetry point. Circular dispersion in reduced $\mathrm{k}$ space means that the medium is an isotropic medium and the wavevector of the acoustic wave and the group velocity are parallel for any propagation direction. Therefore, in the material the velocity of the acoustic wave does not depend on the direction that the wave travels. As can be seen, the radius of the EFCs clearly increases as the frequency increases.

An equi-frequency plot of the 2 nd band, which is a little bit more complicated than the $1^{\text {st }}$ band, which exhibits a square-like contour. The case of a non-circular equi-frequency contour, however, indicates anisotropic behavior. On the other hand, a square like contour also reflects some symmetry in a particular direction. This could be especially advantageous and even be more effective if the self-collimation property of the phononic crystal is considered. It must be noted that the group velocity $\mathrm{v}_{\mathrm{g}}$ is normal to the equi-frequency contour but not collinear to the wave vector $\mathrm{k}$. At each frequency, the energy flow direction is given by the normal to the equi-frequency contour, and it is in the direction of the maximum rate of change of frequencies. The calculated contours also allow us to analyze whether the photonic crystal can have negative refraction or not, depending on the sign of the dispersion slope. From the equi-frequency contour 


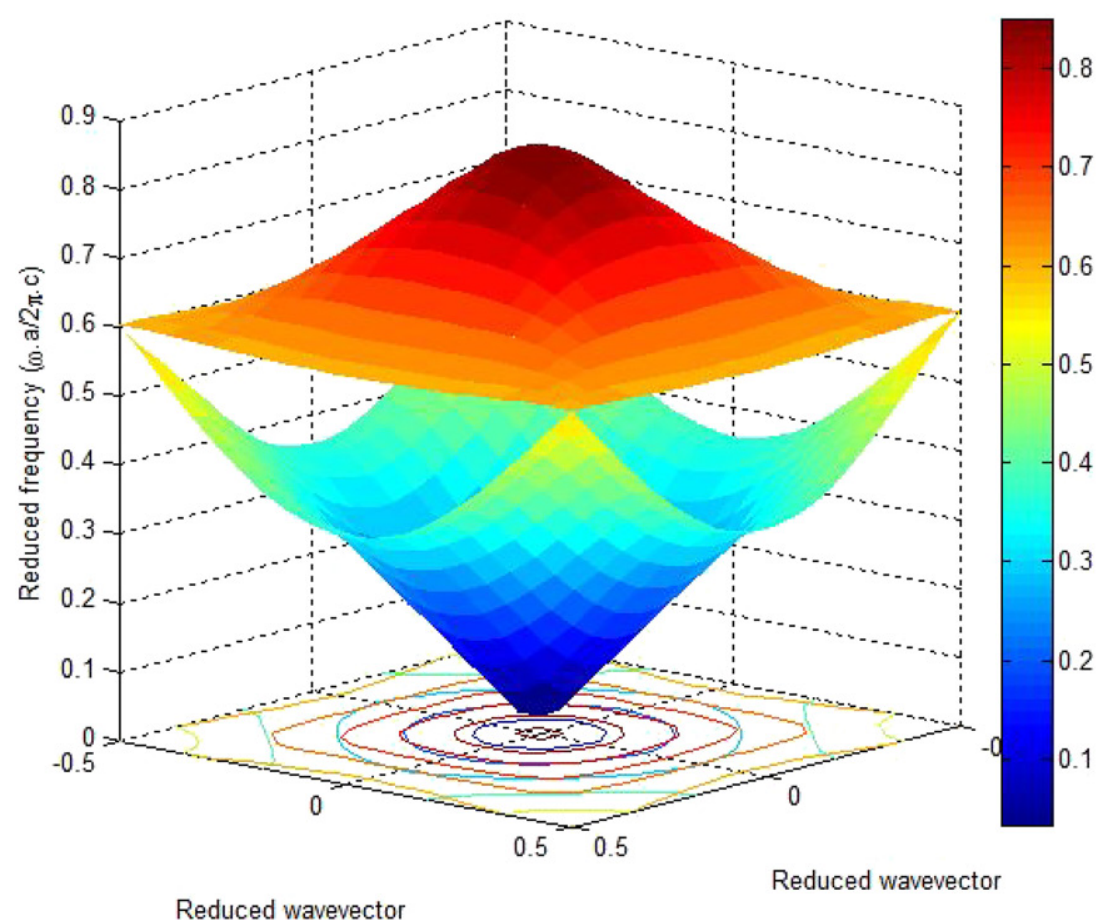

Figure 3. The dispersion, $\omega(\mathrm{k})$, relation (band-diagram) for the first and second bands of the phononic crystal, calculated for all k-vectors in the first Brillouin zone and the equifrequency contours for the first zone.

plots above, we can see that the radius of the circle increases with frequency. Hence, we can conclude that the dispersion slope is positive.

The unusually large angles $\left(\sim 180^{\circ}\right)$ between the phase and group velocities of acoustic waves lead to many unusual wave phenomena observed at crystal-air or crystal-isotropic medium interfaces. In order to understand the dynamics of wave propagation, the concept of group velocity may be useful from the viewpoint of energy transportation. From [2, 9], it follow that the envelope of the wave packet propagates with the velocity $v_{\mathrm{g}}$. The direct calculation of the derivative of the dispersion relation calculated numerically is not always convenient and can give an error. As shown in [2], the group velocity of the wave packet is equal to the velocity of energy transfer. Thus, by using the results of $[2,9]$ the group velocity can always be calculated more accurately, irrespective of the number of points in the dispersion curve. Therefore, we have calculated the group velocity along the high-symmetry directions $\Gamma-\mathrm{X}-\mathrm{M}-\Gamma$ of the Brillouin zone. It is evident that the components of the group velocity versus the high symmetry direction $(\Gamma-\mathrm{X}-\mathrm{M})$ vary over wide limits. The dependences $v_{\mathrm{g}}$, which describe the wave packets of the localized modes of any order exhibit (generally) a maximum at certain propagation constants. This means that the dispersion of group velocity can be positive, negative or zero $[2,9]$. It is noteworthy that the group velocity of the waves is zero at the high symmetric point $\mathrm{X}$ meaning that there is no energy transfer at this point. It is defined as negative refraction that is, the waves refract at the crystal surface in case of the crystal has a negative refractive index. If the band structure of the $\mathrm{PnC}$ has a convex peak at the $\mathrm{X}$ point in Figures 2 and 3, it could result in negative 


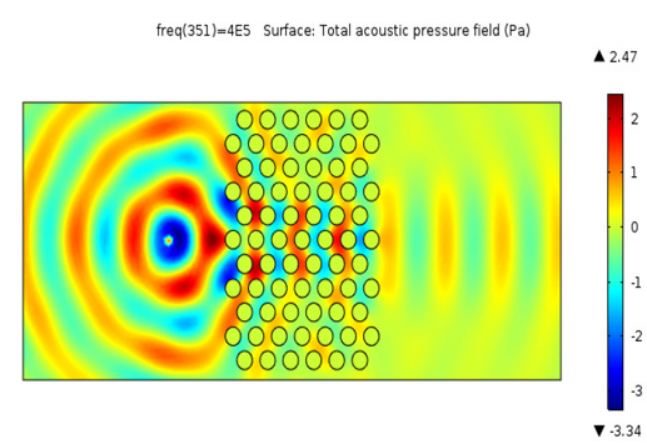

(a)

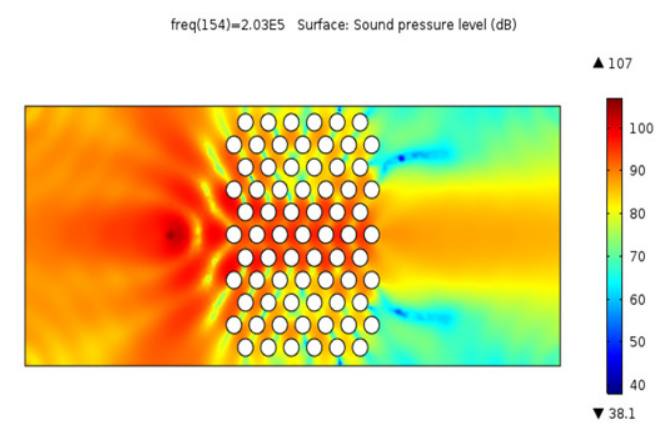

(b)

Figure 4. Acoustic lens effect of $P C$ a) Normalized Total Pressure Field (Pa) b) Sound Pressure Level (dB).

refraction [9]. The negative refraction properties of PC may lead to the possibility of developing flat lenses for focusing acoustic waves. We investigated the acoustic lens effect and negative refraction properties of the PC. PC consists of $\mathrm{LiVCuO}_{4}$ cylindrical inclusion in PVDF. As seen in Figure 4 the PC has negative refraction properties that give a result for the acoustic lens effect.

The PC crystal slab is placed in PVDF and a point source is placed at the left side of the slab. Acoustic waves are emitted from the point source and propagate into the PC slab, which has a negative refractive index. As seen in Figure 4 the transmitted waves are then refocused at the right side of the slab.

\section{Conclusions}

In the present paper, we initially discussed the band structure of two dimensional phononic crystal composed of a multiferroic material embedded in a polymer host. We used the plain wave expansion method to calculate band gaps in the acoustic band structure of the locally resonant phononic crystal. The results of this study illustrate that the width of the first band decreases and more phononic stop bands appear under the assumption of the "solid/solid" approach. The findings of this paper may be useful for achieving improvements in the design of acoustic phononic crystals and they also provide some perspectives a way to design acoustic filters or insulators.

\section{Acknowledgments}

This work is supported by the projects DPT-HAMIT, DPT-FOTON, and NATO-SET-193 as well as TUBITAK under the project nos., 113E331, 109A015, and 109E301. One of the authors (Ekmel Ozbay) also acknowledges partial support from the Turkish Academy of Sciences.

\section{References}

[1] C. M. Soukoulis, Photonic Band Gaps and Localization (Plenum Press, New York, NY, 1993).

[2] R. Craster, and S. Guennau, Elastic, Acoustic and Seismic Metamaterials (World Scientific Handbook of Metamaterials and Plasmonics, Singapore, 2018). 
[3] Y. Pennec et al., Phononic crystals and manipulation of sound, Phys. Status Solidi. 6 (9), 2080 (2009). DOI: 10.1002/pssc.200881760.

[4] M. Maldovan, and E. L. Thomas, Periodic Materials and Interference Lithography: For Photonics, Phononics and Mechanics (Wiley-VCH, Weinheim, 2009).

[5] W. Cheng et al., Observation and tuning of hypersonic bandgaps in colloidal crystals, Nat. Mater. 5 (10), 830 (2006). DOI: 10.1038/nmat1727.

[6] M. M. Sigalas et al., Classical vibrational modes in phononic lattices: theory and experiment, Zeitschrift für Kristallographie - Crystalline Materials. 220 (9-10), 765 (2005).

[7] A. Srivastava, Elastic metamaterials and dynamic homogenization: a review. Int. J. Smart Nano Mater. 6 (1), 41 (2015). DOI: 10.1080/19475411.2015.1017779.

[8] V. A. Burov et al., Acoustic waves in metamaterials, crystals, and anomalously refracting structures, Uspekhi Fizicheskikh Nauk. 181 (11), 1205 (2011).

[9] R. V. Craster, and S. Guenneau, Acoustic Metamaterials: Negative Refraction, Imaging, Lensing and Cloaking (Springer Series in Materials Science, New York, NY, 2013).

[10] M. M. Vopson, Fundamentals of multiferroic materials and their possible applications, Crit. Rev. Solid State Mater. Sci. 40 (4), 223 (2015). DOI: 10.1080/10408436.2014.992584.

[11] J. Seidel (Ed.), Topological Structures in Ferroic Materials: Domain Walls, Vortices and Skyrmions (Sydney Springer, Berlin, 2016).

[12] H. S. Nalwa, Ferroelectrics Polymers: Chemistry, Physics and Applications (Marcel Deccer, New York, NY, 1995). 